\title{
Experiencias vivenciales del personal de enfermería en la campaña de vacunación covid en el cantón Ambato provincia de Tungurahua
}

\author{
Willian Alfredo Llerena Saca \\ wllerena3981@uta.edu.ec \\ https://orcid.org/0000-0003-2753-2624 \\ Lic.Mg. Eulalia Isabel Analuisa Jiménez \\ ei.analuisa@uta.edu.ec \\ https://orcid.org/0000-0002-7906-7640
}

Universidad Técnica de Ambato

Ambato - Ecuador

\section{RESUMEN}

Introducción: La pandemia ha provocado en el mundo una grave crisis sanitaria, social y económica. Exigiendo al Ministerio de Salud Pública del Ecuador, poner en marcha el plan de vacunación COVID, requiriendo que el personal de salud que labora en primera línea sean los principales participantes; produciendo en ellos un cambio en el estilo de vida por las largas jornadas laborales Objetivo: Conocer las experiencias vivenciales del personal de Enfermería que participa en la campaña de vacunación COVID en el cantón Ambato provincia de Tungurahua. Método: Esta investigación tiene un diseño de enfoque cualitativo de tipo exploratorio fenomenológico, con una muestra tomada por conveniencia y razonada, con un total de 12 profesionales en enfermería que poseen el mismo fenómeno de interés, para la recolección de datos se utilizó una entrevista semiestructurada. Resultados: Con las entrevistas realizadas se elaboró una categorización de la información, logrando clasificarlas en: Precepción de la estrategia de la Vacunación COVID, Tipos de experiencias negativas y positivas en la campaña de vacunación, Cambios en el estilo de vida, salud física y mental, Actitudes percibidas en el proceso de vacunación, Relaciones interpersonales. Conclusiones: Las experiencias vividas por parte del personal de enfermería en la campaña de vacunación COVID, en su mayoría fueron experiencias, clasificándolas así: positivas y negativas, de acuerdo con las entrevistas realizadas a cada profesional.

Palabras Clave: vacunación; estilo de vida; experiencias. 


\title{
Living experiences of enfeemria staff in the covid vaccination campaign in the canton Ambato province of Tungurahua
}

\begin{abstract}
Introduction: The pandemic has caused a serious health, social and economic crisis in the world. Demanding the Ministry of Public Health of Ecuador, to implement the COVID, vaccination plan requiring health personnel working on the front line to be the main participants; producing in them a change in lifestyle due to long working hours. Objective: To learn about the experiences of nursing personnel who participate in the COVID vaccination campaign in the Ambato Canton, Province of Tungurahua. Method: The research is a qualitative approach design of phenomenological exploratory type, with a sample taken by convenience and reasoned, with a total of 12 nursing professionals who have the phenomenon of interest, using a semi-structured interview as an instrument for data collection. Results: with the interviews conducted, a categorization of the information was elaborated, managing to classify them in: Perception of the COVID Vaccination strategy, Types of negative and positive experiences in the vaccination campaign, Changes in lifestyle. Physical and mental health, Perceived attitudes in the vaccination process, Interpersonal relationships. Conclusions: The experiences lived by the nursing staff in the COVID vaccination campaign were mostly good experiences, thus classifying them into positive and negative according to the interview conducted with each professional.
\end{abstract}

Key words: vaccination; lifestyle; experiences.

Artículo recibido: 30 noviembre. 2021 Aceptado para publicación: 29 diciembre 2021 Correspondencia: wllerena3981@uta.edu.ec Conflictos de Interés: Ninguna que declarar 
Experiencias vivenciales del personal de...

\section{INTRODUCCIÓN}

Hace más de un año que se declaró la pandemia por coronavirus SARS-CoV-2 que tantos casos y tantas muertes ha causado en todo el mundo. Contabilizando la OMS aproximadamente 117 millones de casos y 2,5 millones de muertes.(Yanez, 2021)

La pandemia ha provocado en el mundo una grave crisis sanitaria, social y económica, mostrando la debilidad de los sistemas sanitarios y de los organismos de salud pública para poder dar una respuesta rápida y adecuada a una situación inesperada. Existiendo además un colapso en el sistema de salud, dificultando la atención a otros tipos de enfermedades. (01-Plan-nacional-de-vacunacion-e-inmuniczacion-contra-el-COVID-19-Ecuador-20211.pdf, s. f.-a)

La actual pandemia ha generado que la comunidad científica de todo el mundo implemente estrategias de prevención y contención del Corona virus, dentro de las cuales se encuentra el lavado de manos que debe durar de 40 a 60 segundos, la desinfección de manos con solución en base alcohol con una duración de 20 a 30 segundos, el uso de mascarillas, el distanciamiento social, incluso la suspensión o limitación de concentraciones multitudinarias, el cierre de lugares de trabajo no esenciales y establecimientos educativos, la reducción del transporte público, y la limitación de los viajes. (01-Plan-nacional-devacunacion-e-inmuniczacion-contra-el-COVID-19-Ecuador-2021-1.pdf, s. f.-a)

El Ministerio de Salud Pública de Ecuador ha implementado un plan de vacunación contra COVID, comenzando la vacunación con los grupos más vulnerables como son el personal de salud y adultos mayores, existiendo una gran demanda de personas que quieren ser inoculadas, exigiendo que el personal de salud que se encuentra laborando en primera línea, sean los partícipes en esta campaña de vacunación. (ientos_para_la_recepciOn_tEcnica_de_vacunas_covid-19_firmado-signed-signedsigned-1-signed_l.pdf, s.f.) Provocando así en ellos cambios en su estilo de vida, relaciones y roles familiares, y afectaciones en su salud; especialmente en el personal de enfermería en el cual está enfocado el presente artículo científico.

Las largas jornadas laborales que el personal de enfermería atraviesa durante la campaña de vacunación contra el COVID, ha provocado adaptarse a cambios en sus horarios de trabajo, comida y descanso, influyendo en muchas ocasiones, cambios en las relaciones y roles familiares. De ahí parte la importancia de la realización de esta investigación, para poder dar a conocer a la comunidad en general el arduo trabajo que el personal de 
enfermería realiza durante la campaña de vacunación. Además, para la realización de esta investigación se está empleando un diseño con enfoque cualitativo de tipo exploratorio fenomenológico, el cual nos permitirá conocer las experiencias vivenciales del personal de enfermería que participa en la campaña de vacunación COVID, en el cantón Ambato, provincia de Tungurahua.(Lévano, 2007)

Finalmente, desde el punto de vista científico, este artículo servirá de referencia para proyectos venideros y así seguir aportando con conocimientos a la población a la cual se dirige dichos estudios.

\section{ESTRATEGIAS METODOLÓGICAS O MATERIALES Y MÉTODOS}

La metodología empleada será mediante un diseño de enfoque cualitativo de tipo exploratorio fenomenológico,(Otzen \& Manterola, 2017) ya que, se pretende evidenciar las experiencias vivenciales del personal de enfermería en la campaña de vacunación, lo cual nos permitirá conocer el estilo de vida, roles familiares y estado salud en el personal de enfermería que participan en la campaña de vacunación COVID en el cantón Ambato, siendo esto un criterio de inclusión para realizar la investigación.

La muestra tomada fue por conveniencia y razonada, con un total de 12 profesionales en enfermería que poseen el fenómeno de interés, tomando en cuenta el criterio de saturación de la información. (Ortega-Bastidas, 2020) Asimismo el tipo de muestreo tiene como características: a) establecer diferentes etapas de selección de muestra. b) identificar sujetos que cuenten con el fenómeno en general.(239035878001.pdf, s. f.)

De la misma manera, para la participación en la investigación se presentó, un consentimiento informado el cual permitió asegurar la participación libre y voluntaria del personal de enfermería, respetando los 4 principios básicos de bioética que son: Beneficencia, No maleficencia, Autonomía y Justicia. (1.pdf, s. f.)

Igualmente, para la publicación de la información se creó un seudónimo para cada participante.

El instrumento utilizado para la recolección de datos es una entrevista semiestructurada en la cual las preguntas están definidas previamente en un cuestionario para una entrevista, pero la secuencia, así como su formulación pueden variar en función de cada sujeto entrevistado. Es decir, el/la investigador/a realiza una serie de preguntas (generalmente abiertas al principio de la entrevista) que definen el área a investigar, pero tiene libertad 
para profundizar en alguna idea que pueda ser relevante.(Técnicas conversacionales para la recogida de datos en investigación cualitativa, s. f.)

Posteriormente, los datos recolectados fueron transcritos de forma digital sin manipulación de la información, para esto se creó una unidad hermenéutica de análisis, aplicando el paquete estadístico para análisis cualitativo atlas T.I. (Soratto et al., 2020) permitiéndonos la codificación y categorización de los datos realizados de acuerdo a los objetivos de la investigación, que nos ayudó con la clasificación de la información, mediante citas textuales que nos permitan la triangulación de la información de una mejor manera. 


\section{RESULTADOS Y DISCUSIÓN}

\begin{tabular}{|c|c|c|c|}
\hline \multicolumn{4}{|l|}{ Categorías } \\
\hline $\begin{array}{l}\text { Seudónimos de los } \\
\text { participantes }\end{array}$ & $\begin{array}{l}\text { Percepción de la estrategia de la } \\
\text { Vacunación COVID. }\end{array}$ & $\begin{array}{c}\text { Tipos de experiencias negativas y } \\
\text { positivas en la campaña de vacunación. }\end{array}$ & Cambios en el estilo de vida. \\
\hline María & $\begin{array}{l}\text { "Fue una decisión muy buena que el } \\
\text { gobierno hayan traído las vacunas para } \\
\text { poder aplicar y poder combatir el COVID" }\end{array}$ & $\begin{array}{l}\text { "Fue muy importante porque si no hubiera } \\
\text { enfermera no hubiera quien administre la } \\
\text { vacuna a la población" }\end{array}$ & $\begin{array}{l}\text { "Se salía bien noche a veces } 9 \text { o } 10 \text { de la noche } \\
\text { entonces fue eso, otras veces tener que hacer } \\
\text { esperar a mi esposo con mis hijos, mis hijos } \\
\text { llorando o durmiendo" }\end{array}$ \\
\hline Lupe & $\begin{array}{l}\text { "Creo que la estrategia utilizada por el } \\
\text { ministerio de salud ha sido muy acertada, ha } \\
\text { sido muy organizada" }\end{array}$ & $\begin{array}{l}\text { "Creo que a pesar de las posibles dificultades } \\
\text { que teníamos siempre queda la satisfacción } \\
\text { que hayamos podido ayudar con un granito } \\
\text { de arena para reducir las muertes por } \\
\text { COVID" }\end{array}$ & $\begin{array}{l}\text { "En ocasiones no he tenido tiempo para } \\
\text { almorzar; unos } 10 \text { a, } 20 \text { minutos y ya } \\
\text { comenzamos a trabajar, creo que esa fue la } \\
\text { parte más complicada" }\end{array}$ \\
\hline Viviana & $\begin{array}{l}\text { "Para mí fue bueno, ya que, como Ud. sabe } \\
\text { tenemos mucha población a quien debemos } \\
\text { vacunar" }\end{array}$ & $\begin{array}{l}\text { "Fue muy importantes ya que cada quien } \\
\text { cumplió una pequeña parte en el proceso de } \\
\text { vacunación" }\end{array}$ & $\begin{array}{l}\text { "Salíamos muy tarde y ya en casa toda la } \\
\text { familia, se iba a acostar", }\end{array}$ \\
\hline Teresa & $\begin{array}{l}\text { "Desde mi punto de vista es muchas } \\
\text { matrices, muchos papeles, mucho trabajo } \\
\text { para una sola persona" }\end{array}$ & $\begin{array}{l}\text { "La participación hace que demos mayor } \\
\text { tiempo a la institución en la cual a nosotros } \\
\text { ni siquiera nos reconocen el tiempo de extra } \\
\text { trabajo" }\end{array}$ & $\begin{array}{l}\text { "Que hemos dejado a un lado a nuestras } \\
\text { familias a pesar en que nuestro corazón } \\
\text { siempre ha estado el temor a contagiarnos" }\end{array}$ \\
\hline Rosa & $\begin{array}{l}\text { "Bueno la estrategia que han emitido es muy } \\
\text { buena y fundamental para la reducción de } \\
\text { casos de COVID" }\end{array}$ & $\begin{array}{l}\text { "El trabajo fue muy arduo y seguimos en la } \\
\text { lucha, hasta el momento seguimos } \\
\text { realizando esta actividad." }\end{array}$ & $\begin{array}{l}\text { "Sí nos afectó bastante a todas las familias, } \\
\text { amigos" }\end{array}$ \\
\hline Pamela & $\begin{array}{l}\text { "Se consideró grupos prioritarios el personal } \\
\text { que estamos en primera línea a adultos } \\
\text { mayores y personas con discapacidad, } \\
\text { porque ellos son los más vulnerables para el } \\
\text { contagio" }\end{array}$ & $\begin{array}{l}\text { "Horas excesivas de trabajo ya que } \\
\text { iniciábamos } 7: 30 \text { de la mañana y no } \\
\text { teníamos hora de salida entonces fue muy } \\
\text { agobiante" }\end{array}$ & $\begin{array}{l}\text { "Mi estilo de vida cambió radicalmente, ya } \\
\text { que, teníamos que entrar antes de las } 8 \text { para ya } \\
\text { tener todo listo y salir muy tarde dejar } \\
\text { abandonado a mis hijos" }\end{array}$ \\
\hline
\end{tabular}




\begin{tabular}{|c|c|}
\hline Carlos & $\begin{array}{l}\text { "La perspectiva que tuve y al ser parte de } \\
\text { ésta fue muy buena, en vista que se pudo } \\
\text { vacunar grupos vulnerables" }\end{array}$ \\
\hline Carla & $\begin{array}{l}\text { "Se ha llevado una buena organización } \\
\text { por parte del Ministerio de Salud } \\
\text { Pública." }\end{array}$ \\
\hline Renzo & $\begin{array}{l}\text { "Me parece bastante acertada, porque } \\
\text { prácticamente se trabajó por grupos de } \\
\text { edad" }\end{array}$ \\
\hline Natalia & $\begin{array}{l}\text { "Pues es una estrategia organizada y } \\
\text { planteada por ellos, para poner por } \\
\text { grupos de edad" }\end{array}$ \\
\hline Ibeth & $\begin{array}{l}\text { "Fue un poco sobresaltada porque toda la } \\
\text { población quería ser inmunizado y el } \\
\text { personal solicitarlo fue poco capacitado" }\end{array}$ \\
\hline Leonardo & $\begin{array}{l}\text { "Bueno en este caso sería mitigar o } \\
\text { minimizar la sintomatología COVID en } \\
\text { su mayoría" }\end{array}$ \\
\hline
\end{tabular}

"Al ser parte del plan de vacunación me siento muy orgulloso, porque formé parte de este gran grupo de personas"

"Considero que ha sido una participación positiva, puesto que, hemos logrado vacunar e inmunizar a la mayoría de pacientes"

"Profesionalmente es un reto enorme, porque el estar al frente de la inmunización de Ambato, es un desafío tener que estar coordinando absolutamente todo"

"Ha sido una clave importante para el proceso de vacunación, ya que, sin nosotros no hubiera avanzado la campaña de vacunación"

"Hubo mucha sobrecarga en el trabajo, la carga horaria, el cansancio, incluso algunas jornadas con mucha población en los centros de vacunación"

"Yo considero bastante activa nuestra participación, no solo en los días laborables también en otros días extras"
"Por motivos de trabajo, tuve que trasladarme a otra provincia estuvo más complicada, más en cuanto a mi familia se refiere"

"No he tenido una experiencia negativa en cuanto a mi estilo de vida"

"Fue un cambio brusco desde la parte profesional, porque tocó adecuarnos a una nueva forma de trabajo"

"Yo creo que el cansancio físico y mental que se pudo presenciar es porque fue muy complicado para muchas personas".

"Al inicio fue una emoción, pero ya después era un poco estresante, era mucho el estrés con la presión que teníamos de cumplir"

"El estrés cansancio físico, emocional, de pronto no poder contar el $100 \%$ con nuestras familias"

Gráfico: 1

Fuente: Entrevistados - Elaborado: Autores 
Categorías

Seudónimos de

los participantes

María "Hubo personas que nos trataba mal, nos estábamos vacunando a nuestra conveniencia"

"Nos toca estar paradas, he tenido dolores musculares, ese tipo de cosas siempre van a estar presente por el hecho mismo de ser un trabajo bastante fuerte"

"Se terminaba muerta, cansada, estresados porque no cuadraba los datos y otras tantas cosas"

Viviana

"Emocionalmente mucho ya que desde que me enfermé de COVID yo pensé que voy a morir, tenía mis hijas que aún están bajo mi poder"

Teresa

Rosa y eso que estamos al momento con grupo de adultos mayores"
"Si el estrés fundamental es una afectación

\section{Actitudes percibidas en el proceso de}

\section{vacunación}

"El trato de las personas al comienzo no fue bueno, la gente quería que les vacunen como ellos querían"

"Los pacientes al momento que están molestos; uno como enfermera trata de calmarlos y después proceder a la vacunación"

"Muchos pacientes aceptaron la vacuna de buena manera; como le digo aceptaron la vacuna y que teníamos disponibilidad de muy buena manera, y otros pacientes pues no"

"La actitud de los pacientes era que tenían miedo, porque están poniendo cosas que tal vez les van a eliminar a los usuarios, vayan a morir, pero les explicábamos que cualquier vacuna que les apliquemos todas son efectivas"

"Bueno ellos sentían temor en la aplicación, porque igual las vivencias que ellos tenían fueron de tristeza, porque ellos habían perdido a sus familias"

\section{Relaciones interpersonales}

"Hay personas que nos trata mal, van hablando no a todos les caemos bien"

“Desde el primer día de mí parte siempre he tratado de mantener una buena relación con el paciente y ser más cordial"

"Muchos pacientes aceptaron la vacuna de buena manera; como le digo aceptaron la vacuna y que teníamos disponibilidad de muy buena manera, y otros pacientes pues no"

"A pesar que estaba emocionalmente mal trataba de darle una mejor impresión de mí y atenderle de una mejor manera"

"En la relación siempre debemos mantener esa cordialidad, demostrando educación al paciente mismo referente a la vacuna" 


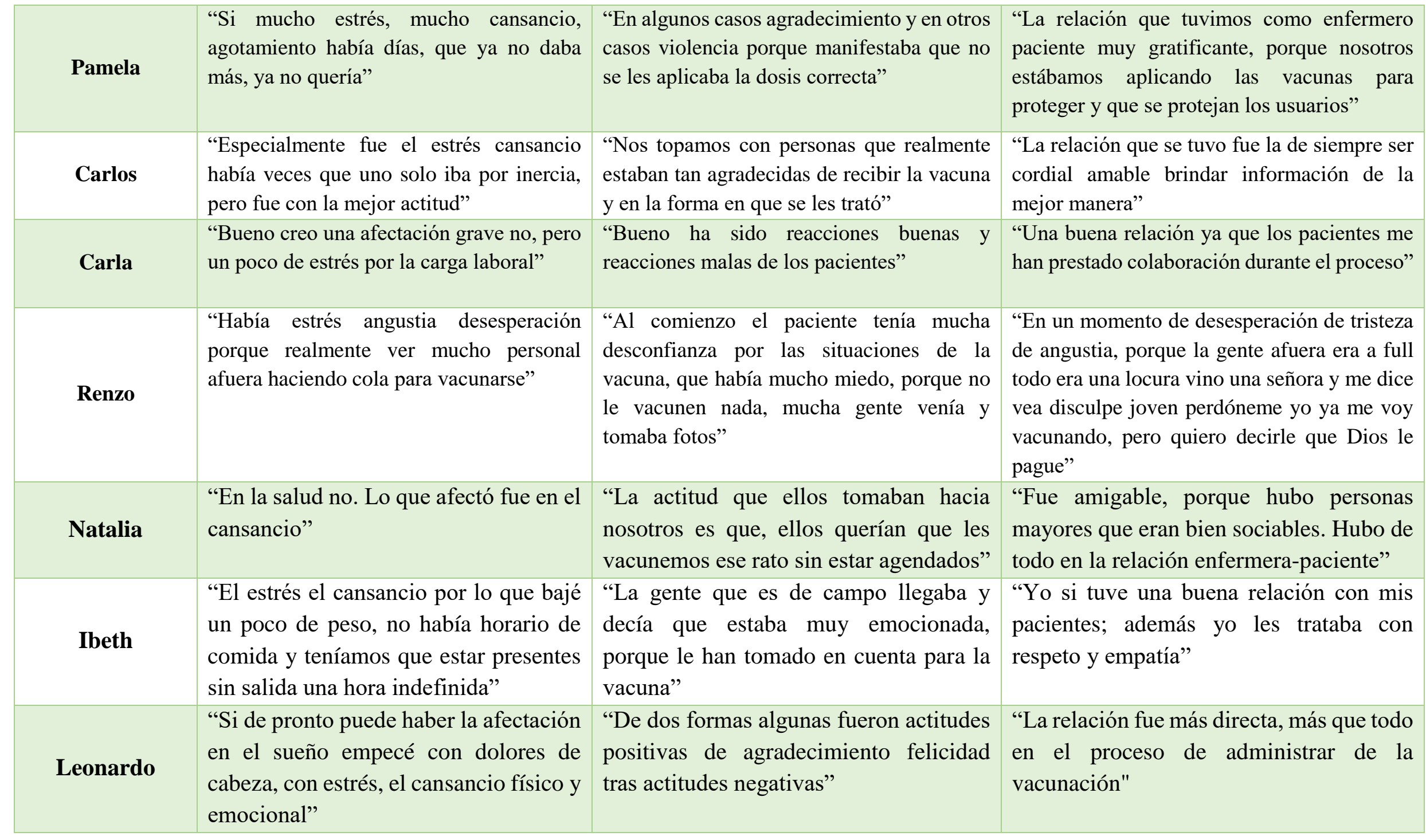

Tabla: 2

Fuente: Entrevistados - Elaborado: Autores 
El Ministerio de Salud Pública de Ecuador con la programación de la vacunación COVID dio sus inicios con, grupos poblacionales con riesgo significativamente elevado de sufrir un cuadro grave o la muerte. (01-Plan-nacional-de-vacunacion-e-inmuniczacion-contrael-COVID-19-Ecuador-2021-1.pdf, s. f.-a)

Con el propósito de contribuir con la inmunidad del país, es necesario vacunar, al menos al 70\% de la población de cada provincia, en cumplimiento de la recomendación técnica internacional de vacunación de rebaño. Para este fin, el Gobierno ecuatoriano ha adelantado estrategias para la adquisición de vacunas suficientes para la inmunización de la población y lograr vacunar al $86 \%$ de la población objetivo del Ecuador.(01-Plannacional-de-vacunacion-e-inmuniczacion-contra-el-COVID-19-Ecuador-2021-1.pdf, s. f.-b)

Como el plan de vacunación ha beneficiado a toda la población en general, así mismo ha presentado un gran reto en el personal de enfermería al no estar técnicamente capacitado en el ámbito administrativo, para llevar la contabilidad exacta de cientos de personas que tenían que ser inoculadas por día en cada puesto de vacunación, creando un gran malestar en el personal de enfermería al tener que llevar a su cargo las matrices de la vacunación COVID, pero al mismo tiempo para la mayoría de profesionales es una gran satisfacción ser parte de la campaña de vacunación y ayudar a mitigar esta enfermedad.

De la misma manera, la participación del personal de enfermería en el plan de vacunación ha demostrado las fortalezas de cada profesional, siendo un pilar muy importante el personal de enfermería, ya que, con los conocimientos adquiridos durante su vida profesional en el ámbito de la salud pública, ayudando a poner en marcha de una manera efectiva el plan de vacunación.

Sin embargo, el personal de enfermería ha presentado mayor exigencia en su campo laboral, sobrepasando así su carga horaria de trabajo incluso sin ser reconocidos por su tiempo y esfuerzo en la campaña de campaña de vacunación.

Igualmente, la sobre carga laboral ha provocado que los profesionales no puedan compartir tiempo con su familia, esto nos cuenta una de las personas entrevistadas.

"Mi estilo de vida cambió radicalmente, en vista que teníamos que entrar antes de las 8 para ya tener todo listo y salir muy tarde dejar abandonado a mis hijos" (Pamela)

La influencia negativa del trabajo en la familia, denominada conflicto trabajo-familia, puede estar asociado porque el tiempo y las energías destinadas al trabajo son mayores, 
causando discordancia entre el tiempo dedicado a la familia y al trabajo. (López-Andrade et al., 2021)

El personal de enfermería ha presentado un cambio en su estilo de vida y especialmente en su rol familiar, llegando incluso a no pasar tiempo en sus hogares con sus familias y dedicando mayor tiempo a su campo laboral.

Además, los hábitos alimenticios es una base fundamental en el mantenimiento de la salud, tanto a nivel individual como poblacional.(Herruzo Caro et al., 2017)

Por lo tanto, en muchas ocasiones los profesionales de la salud no presentan una dieta y hora adecuada para sus comidas, llegando a producirse en muchas ocasiones problemas en su estado de salud a futuro. Esto nos cuenta una profesional entrevistada.

"A veces no he tenido tiempo de almorzar, unos 10 a 20 minutos y ya comenzamos a trabajar creo que esa ha sido la parte más complicada" (Lupe)

Los hábitos alimentarios de una población, dependen de su cultura, sus creencias y sus costumbres, determinando su estado de salud, ya que, algunos son perjudiciales; porque nos hacen más susceptibles a la aparición de enfermedades. Mientras, que otros son beneficiosos, porque promueven un mejor estado de salud.(Manriquez et al., s. f.)

El trabajo en la vacunación, ha forjado en el profesional de enfermería, no solo presenta un cambio en su estilo vida, sino también en su forma de trabajo y en las metas propuestas en las actividades intramurales que también deben cumplir en su centro de salud, provocando estrés en el profesional.

El estrés aparece cuando los recursos con los que se cuenta resultan insuficientes ante las exigencias del entorno. Ese estado psicológico puede provocar efectos no experimentados anteriormente por el individuo, hasta que se ve precisado a enfrentar metas que comprometen su estatus social y laboral sin las condiciones propicias, lo que causa un desequilibrio en su condición física y mental (Delgado Espinoza et al., 2020). Contado esto por un profesional entrevistado.

"Especialmente fue el estrés, cansancio; había veces que uno solo iba por inercia, pero fue con la mejor actitud" (Carlos)

El excesivo trabajo que el personal de enfermería realiza, ha provocado que las afectaciones en su equilibrio de salud mental y física se encuentre afectado provocando en ellos, estrés laboral, agotamiento físico, angustia y desesperación por culminar su jornada laboral. 
De igual importancia las actitudes de las personas que querían ser inmunizadas en ese momento, está relacionada con la desesperación por conseguir una dosis para poder así crear defensas contra una enfermedad que ha matado a miles de personas en el mundo.

Actitudes que han provocado mal trato en el personal de enfermería, por conseguir una dosis, pero asimismo existían personas que llegaban con actitudes de agradecimiento, siendo un aliento en esos momentos difíciles para el personal de enfermería. Relatando lo siguiente este profesional.

"En un momento de desesperación, de tristeza, de angustia; porque la gente afuera era a full todo era una locura, pero vino una señora y me dice: vea disculpe joven perdóneme yo ya me voy vacunando, pero quiero decirle que Dios le pague" (Renzo)

Las relaciones interpersonales son una interacción entre personas, donde las principales características que resaltan son la confianza y la credibilidad en el otro, a través del trato mutuo, respeto, empatía y cordialidad; surgiendo de este modo relaciones amistosas y demostraciones de confidencialidad. ( «RELACIONES INTERPERSONALES ENTRE ENFERMEROS PARA SU BIENESTAR - Aladefe», s. f.)

Teniendo en cuenta las múltiples actitudes que el personal de enfermería presenció, siempre demostraron mantener una buena relación enfermero-paciente, realizando su trabajo con calidad y calidez, mostrando un buena actitud e impresión a pesar del agotamiento físico y mental que presentaban. Narrando lo siguiente este profesional.

"Yo si tuve una buena relación entre mi persona y con mis pacientes, además yo les trataba con respeto y empatía" (Ibeth)

La enfermera ayuda al paciente a satisfacer sus necesidades a través de una relación de cooperación y participación activa, asignando el paciente a la enfermera un papel de sustituto. La enfermera, con su actitud, provoca en el paciente sentimientos similares a los generados en una relación anterior.(Elers Mastrapa \& Gibert Lamadrid, 2016)

\section{CONCLUSIÓN O CONSIDERACIONES FINALES}

Primera: Mediante la entrevista semiestructurada que se aplicó al personal de enfermería se logró conocer las experiencias vivenciales en la campaña de vacunación COVID, en el cantón Ambato provincia de Tungurahua.

Segunda: Las experiencias vividas por parte del personal de enfermería en la campaña de vacunación COVID, en su mayoría fueron experiencias; clasificándolas en positivas y negativas de acuerdo a la entrevista realizada a cada profesional. 
Tercera: Los principales factores que influyeron para que se presenten los cambios en el estilo de vida del profesional, fueron horas excesivas de trabajo, laborar en sus días libre y no poder pasar tiempo con sus familias.

\section{LISTA DE REFERENCIAS}

1.pdf. (s.f.). Recuperado 5 de noviembre de 2021, de https://archivos.juridicas.unam.mx/www/bjv/libros/13/6006/1.pdf

01-Plan-nacional-de-vacunacion-e-inmuniczacion-contra-el-COVID-19-Ecuador-2021-

1.pdf. (s.f.-a). Recuperado 22 de octubre de 2021, de https://www.salud.gob.ec/wp-content/uploads/2021/05/01-Plan-nacional-devacunacion-e-inmuniczacion-contra-el-COVID-19-Ecuador-2021-1.pdf

01-Plan-nacional-de-vacunacion-e-inmuniczacion-contra-el-COVID-19-Ecuador-2021-

1.pdf. (s.f.-b). Recuperado 22 de noviembre de 2021, de https://www.salud.gob.ec/wp-content/uploads/2021/05/01-Plan-nacional-devacunacion-e-inmuniczacion-contra-el-COVID-19-Ecuador-2021-1.pdf

239035878001.pdf. (s.f.). Recuperado 12 de noviembre de 2021, de https://www.redalyc.org/pdf/2390/239035878001.pdf

Delgado Espinoza, S. K., Calvanapón Alva, F. A., Cárdenas Rodríguez, K., Delgado Espinoza, S. K., Calvanapón Alva, F. A., \& Cárdenas Rodríguez, K. (2020). El estrés y desempeño laboral de los colaboradores de una red de salud. Revista Eugenio Espejo, 14(2), 11-18. https://doi.org/10.37135/ee.04.09.03

Elers Mastrapa, Y., \& Gibert Lamadrid, M. del P. (2016). Relación enfermera-paciente: Una perspectiva desde las teorías de las relaciones interpersonales. Revista Cubana de Enfermería, 32(4), 0-0.

Herruzo Caro, B., Martín García, J. J., Molina Recio, G., Romero Saldaña, M., Sanz Pérez, J. J., Moreno Rojas, R., Herruzo Caro, B., Martín García, J. J., Molina Recio, G., Romero Saldaña, M., Sanz Pérez, J. J., \& Moreno Rojas, R. (2017). Promoción de la salud en el lugar de trabajo. Hábitos de vida saludable y factores de riesgo cardiovascular en trabajadores de ámbito sanitario en atención primaria. Revista de la Asociación Española de Especialistas en Medicina del Trabajo, 26(1), 9-21.

Ientos_para_la_recepciOn_tEcnica_de_vacunas_covid-19_firmado-signed-signedsigned-1-signed_1.pdf. (s.f.). Recuperado 22 de octubre de 2021, de 
https://www.hgdz.gob.ec/wp-

content/uploads/2021/02/ientos_para_la_recepciOn_tEcnica_de_vacunas_covid19_firmado-signed-signed-signed-1-signed_1.pdf

Lévano, A. C. S. (2007). INVESTIGACIÓN CUALITATIVA: DISEÑOS, EVALUACIÓN DEL RIGOR METODOLÓGICO Y RETOS. 9.

López-Andrade, M. C., Guamán-Álvarez, A. L., Caguana-Chicayza, L. M., PadillaNarváez, T. P., \& Aguaiza-Pichazaca, E. (2021). Desintegración familiar y factores psicosociales en estudiantes de enfermería. Dominio de las Ciencias, 7(2), 551-567.

Manriquez, N., Silveira, N., \& Velasquez, D. (s. f.). Hábitos alimenticios del personal de enfermería del Hospital Eva Perón y Dr. Carlos Bocalandro. 36.

Ortega-Bastidas, J. (2020). ¿CÓMO SATURAMOS LOS DATOS? UNA PROPUESTA ANALÍTICA “DESDE” Y “PARA” LA INVESTIGACIÓN CUALITATIVA. 45, 7.

Otzen, T., \& Manterola, C. (2017). Técnicas de Muestreo sobre una Población a Estudio. International Journal of Morphology, 35(1), 227-232. https://doi.org/10.4067/S0717-95022017000100037

\section{RELACIONES INTERPERSONALES ENTRE ENFERMEROS PARA SU} BIENESTAR - Aladefe. (s. f.). Enfermería21. Recuperado 14 de noviembre de 2021, de https://www.enfermeria21.com/revistas/aladefe/articulo/316/relacionesinterpersonales-entre-enfermeros-para-su-bienestar/

Soratto, J., Pires, D. E. P. de, \& Friese, S. (2020). Thematic content analysis using ATLAS.ti software: Potentialities for researchs in health. Revista Brasileira de Enfermagem, 73. https://doi.org/10.1590/0034-7167-2019-0250

Técnicas conversacionales para la recogida de datos en investigación cualitativa: La entrevista (I) | NURE Investigación. (s. f.). Recuperado 12 de noviembre de 2021, de https://www.nureinvestigacion.es/OJS/index.php/nure/article/view/408

Yanez, R. J. V. (2021). Estrés percibido asociado a la pandemia por COVID-19 en la ciudad de Guayaquil, Ecuador. 9. 